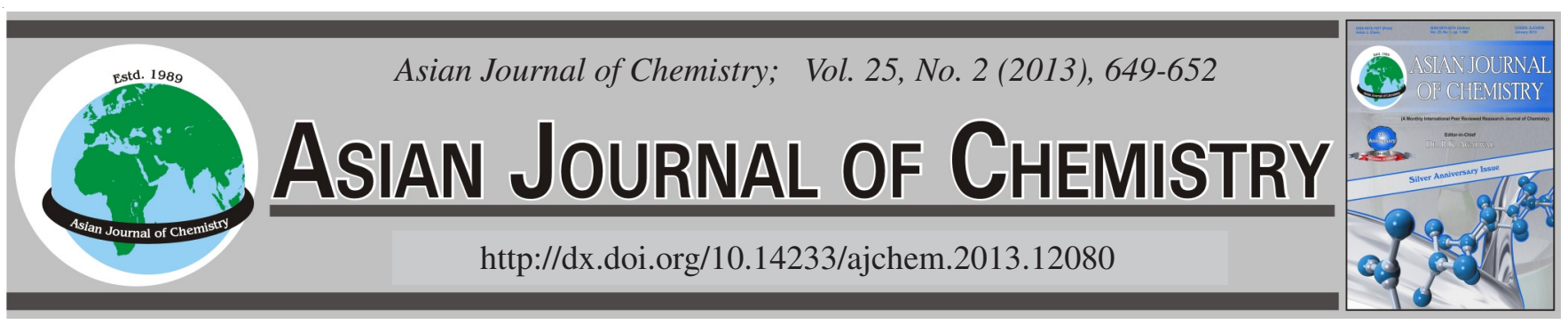

\title{
Facile Synthesis of Novel PbS Dendritic Nanostructures with Tetraphenylphosphonium Bromide as a Ligand via Simple Hydrothermal Process
}

\author{
Xiang-Hu Gao, Xin Zhao, Qing-Fen Geng and Gang Liu*
}

Research \& Development Center for Eco-material and Eco-chemistry, Lanzhou Institute of Chemical Physics, Chinese Academy of Sciences, Lanzhou 730000, P.R. China

*Corresponding author: Fax: +86 931 8277088; Tel: +86 931 4968866; E-mail: gangliu@ licp.cas.cn

\begin{abstract}
Well-defined $\mathrm{PbS}$ dendritic nanostructures were prepared with tetraphenylphosphonium bromide as a ligand via simple hydrothermal process. The Pbs dendritic nanostructures were characterized by X-ray diffraction, (high-resolution) transmission electron microscopy and scanning electron microscopy images. Structural characterization indicates individual $\mathrm{PbS}$ dendritic have three-dimensional structures with one trunk (long axis) and four branches (short axis). The nanorods in each branch are parallel to each other and in the same plane and are perpendicular to the trunk.
\end{abstract}

Key Words: PbS, Tetraphenylphosphonium bromide, Hydrothermal, Dendritic.

\section{INTRODUCTION}

Nanometer-scale materials have attracted considerable interest in recent years due to their unique physical and chemical properties and potential applications in nanoscale devices ${ }^{1}$. Semiconductor nanocrystals have been extensively studied due to their vital applications in numerous technological areas such as microelectronics, electro-optics, non-linear optics, light energy conversion, photocatalysis, photoelectrochemistry and biological fluorescence labeling ${ }^{2}$. Such applications are attributed to their quantum confinement effects, optical, electrical and chemical properties, which are drastically different from the bulk ${ }^{3}$. As a family member of lead chalcogenides, $\mathrm{PbS}$ is an important semiconductor $(\pi-\pi)$ with a small band gap $(0.41$ $\mathrm{eV})$ and an exciton of a large Bohr radius $(18 \mathrm{~nm})^{4}$. Moreover, quantum size $\mathrm{PbS}$ has exceptional third-order non-linear optical properties, which means it should be useful on optical devices such as optical switches. PbS quantum dots with stable efficient luminescence in the near-IR spectral range has potential application in communication, biological imaging and infrared photodetector.

$\mathrm{PbS}$ nanocrystals with different morphologies have been prepared including spheres, cubes, rodlike shapes, tubes, wires, truncated octahedrons and dendritic, star-shaped and flowershaped structures ${ }^{5-10}$. Different synthetic routes such as screw dislocations, microwave irradiation, block copolymer nanoreactors, polydiacetylene langmuir film, mesoporous silica SBA-15 phase, self-assembly, sonochemical synthesis have been employed for the fabrication of $\mathrm{PbS}$ nanoparticles ${ }^{11-15}$. It has been reported that the synthesis of various morphologies of $\mathrm{PbS}$ nanocrystal by using cetyltrimethylammonium bromide (CTAB), sodium dodecyl sulfate (SDS), their mixtures and poly(vinylpyrrolidone) (PVP), L-cysteine, oleic acid and tri$n$-octylphosphine (TOP) ${ }^{16-19}$. They have mentioned that a precise control of surfactant concentration governs the overall morphology of $\mathrm{PbS}$ nanocrystal. According to their results, the surfactant molecules prefer to adsorb at the $\{111\}$ facets due to the presence of a greater charge density in comparison to $\{100\}$. This absorption promotes the growth at $\{100\}$ facets of fcc geometry and results in the formation of various morphologies ${ }^{16}$. Thus, designing and developing new ligands is one of the challenging issues to synthesize novel architectures based on semiconductor nanocrystal. In the present study, novel $\mathrm{PbS}$ dendritic nanostructures was prepared with tetraphenylphosphonium bromide (TPPB) as a ligand via facile hydrothermal process. To the best of our knowledge, this is the first time that TPPB was used as a ligand to prepare $\mathrm{PbS}$ semiconductor nanocrystal.

\section{EXPERIMENTAL}

Thiourea was purchased from Shanghai chemical reagent factory. $\mathrm{Pb}\left(\mathrm{CH}_{3} \mathrm{COO}\right)_{2} \cdot 3 \mathrm{H}_{2} \mathrm{O}$ was purchased from Beijing hongxing Chemical reagent factory. Tetraphenylphosphonium bromide was purchased from Zhejiang Xianju chemical reagent factory. All chemicals were analytical pure and used without further purification. In a typical synthesis process, $1 \mathrm{~g}$ 
TPPB was dissolved in $34 \mathrm{~mL}$ distilled water to form a homogeneous solution, then $0.379 \mathrm{~g}(0.001 \mathrm{~mol}) \mathrm{Pb}\left(\mathrm{CH}_{3} \mathrm{COO}\right)_{2} \cdot 3 \mathrm{H}_{2} \mathrm{O}$ and $0.228 \mathrm{~g}(0.003 \mathrm{~mol})$ thiourea were added into the TPPB aqueous solution. The mixture was stirred vigorously for 40 min with a stirring rate $450 \mathrm{r} / \mathrm{min}$. Finally, the mixture was transferred and sealed in a teflon-lined stainless-steel autoclave of $100 \mathrm{~mL}$ capacity. The autoclave was transferred into vacuum oven and maintained at $120{ }^{\circ} \mathrm{C}$ for $24 \mathrm{~h}$ and allowed to cool naturally to room temperature. The resulting black product was collected by centrifugation, washed several times with water and ethanol and dried at $60{ }^{\circ} \mathrm{C}$.

Characterization: The morphology of $\mathrm{PbS}$ semiconductor nanocrystal was studied by scanning electron microscopy (SEM) using a Field Emission Scanning Electron Microscope (JSM-6701F) with gold sputtered coated films. The chemical composition of the products was recorded by energy dispersive spectroscopy (EDS) equipped in SEM. The structural information on the samples was collected by powder X-ray diffraction (XRD) method. Wide angle X-ray diffraction (WXRD) with a Rigaku D/max-2400 diffractometer $\left(\mathrm{CuK}_{\alpha}\right.$ radiation $=0.15$ $\mathrm{nm}$, generator voltage $=40 \mathrm{kV}$, current $=60 \mathrm{~mA}$ ) was used in this work. Transmission electron microscopy (TEM), electron diffraction (ED) and high-resolution transmission electron microscopy (HRTEM) images were obtained with a Hitachi HF-2010 transmission electron microscope.

\section{RESULTS AND DISCUSSION}

The physical and chemical properties of semiconductor nanocrystals strongly depend not only on the chemical compositions but on particle sizes, surface chemistry, crystalline structures and the presence of defects ${ }^{20}$. The morphology of the prepared $\mathrm{PbS}$ semiconductor nanocrystal was visualized by scanning electron microscopy. Examining numerous SEM images of the sample prepared at $120^{\circ} \mathrm{C}$ for $24 \mathrm{~h}$, we found that almost all the particles are dendrites (Fig. 1a). It indicates that well-defined $\mathrm{PbS}$ dendritic nanostructures can be obtained under present conditions. It is worth to note that the individual $\mathrm{PbS}$ dendritic have three-dimensional structures with one trunk (long axis) and four branches (short axis). The nanorods in each branch are parallel to each other and in the same plane and are perpendicular to the trunk. A higher magnification SEM images can clearly show their 3D structures (Fig. 1b). More interestingly, three dendrites can connect with each other to form a "T" structure, as is clearly shown in Fig. 1c and four dendrites can form a "+" more complex structure, as is clearly shown in Fig. 1d. It can be seen from Fig. 1c that the length of the trunk and the diameter of the branches of the $\mathrm{PbS}$ dendrites is about $3.53 \mu \mathrm{m}$ and $40-100 \mathrm{~nm}$, respectively.

Fig. 2 shows the EDX spectrum taken from an ensemble of nanocrystals. Peaks associated with $\mathrm{Pb}$ and $\mathrm{S}$ are clearly observed and provide strong evidence that the nanocrystals are composed of $\mathrm{PbS}$.

The phase and purity of the as-obtained product is characterized by XRD analysis (Fig. 3). The diffraction patterns distinctly indicate the fine crystallinity of the obtained samples. The reflection peaks of the different products can be indexed to the rock-salt-structured $\mathrm{PbS}$ with a lattice constant of $\mathrm{a}=$ $5.9348 \AA$, which is in good agreement with the literature values
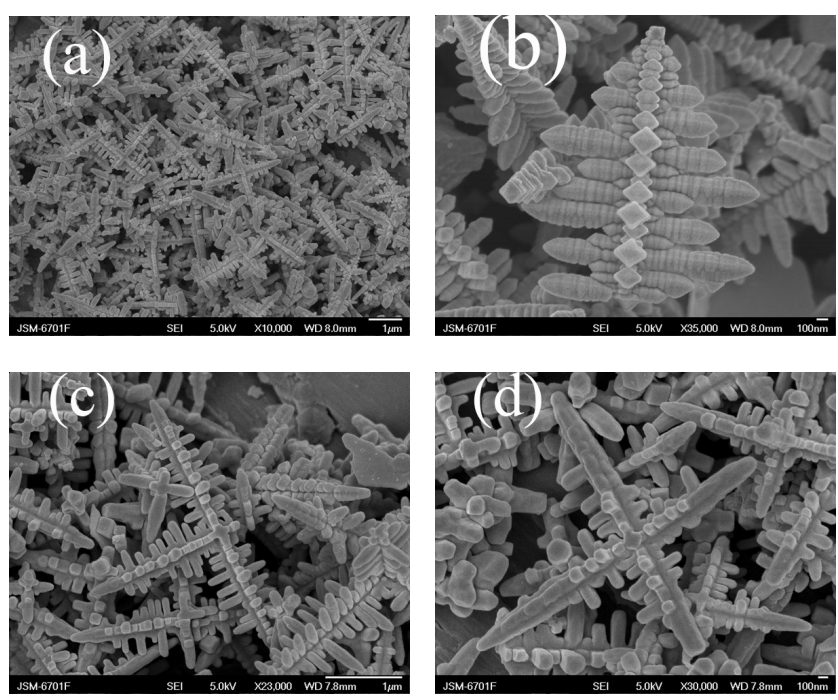

Fig. 1. SEM images of the $\mathrm{PbS}$ dendritic nanostructures prepared by the hydrothermal method. (a) Low-magnification SEM image showing $\mathrm{PbS}$ dendritic nanostructures. (b) Higher magnification SEM image showing their 3D dendritic structure. (c) SEM image of the three or four dendrites connecting to form a "T" type structure. (d) SEM image of the four dendrites connecting to form a "+" structure

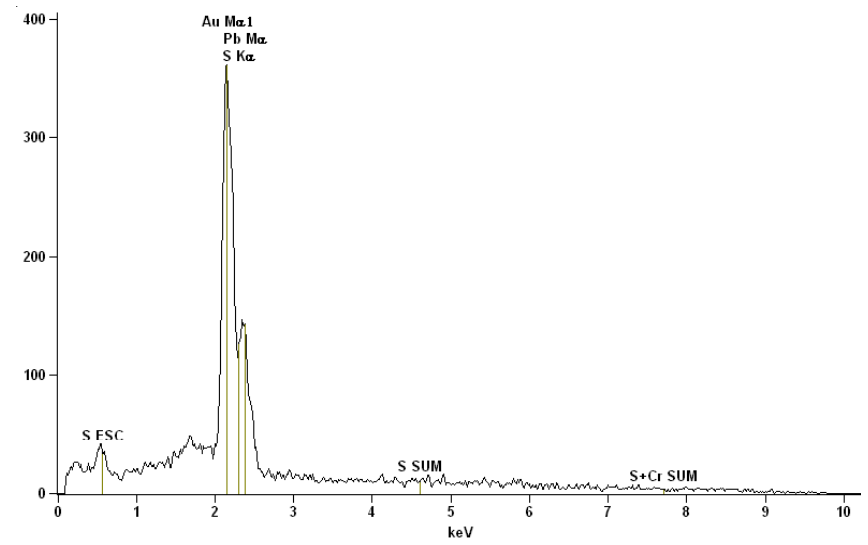

Fig. 2. EDX spectrum of the $\mathrm{PbS}$ dendritic nanostructures

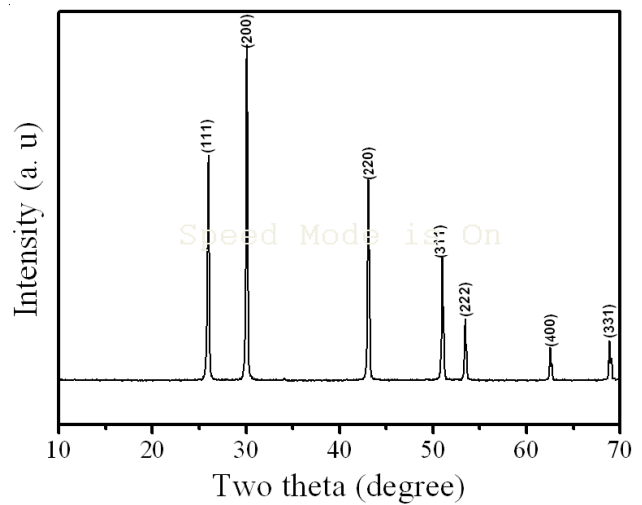

Fig. 3. XRD pattern of the as-synthesized $\mathrm{PbS}$ dendritic

(JCPDS Card No. 5-592). No peaks of impurities were detected, revealing the high purity of the as-synthesized products. It is worth to note that the ratio between the intensities of the (200) and (111) diffraction peaks is somewhat higher than the conventional value (1.30 versus 1.19 ), which indicates that our products may be abundant in $\{100\}$ facets and thus lead to relatively greater accelerated growth along the $<100$ ) $>$ directions. 
The morphology and structure of the $\mathrm{PbS}$ nanopropeller arrays were further confirmed by TEM and selected-area electron diffraction (SAED). Fig. 4a shows a typical TEM image of $\mathrm{PbS}$ dendritic nanostructures. It is worth to note from the Fig. $4 b$ the formation of crosslike "+" PbS dendritic nanostructures. The single-crystalline nature of the $\mathrm{PbS}$ propellers was further revealed by selected area electron diffraction (SAED). SAED patterns recorded from the left branch, the trunk and the right branch in a single propeller are displayed in Fig. 3d-f, revealing that the SAED patterns are almost the same and were all recorded along the $\left[011^{-}\right]$zone axis of the cubic phase of $\mathrm{PbS}$. These patterns further confirm the single crystallinity of the as-prepared products.
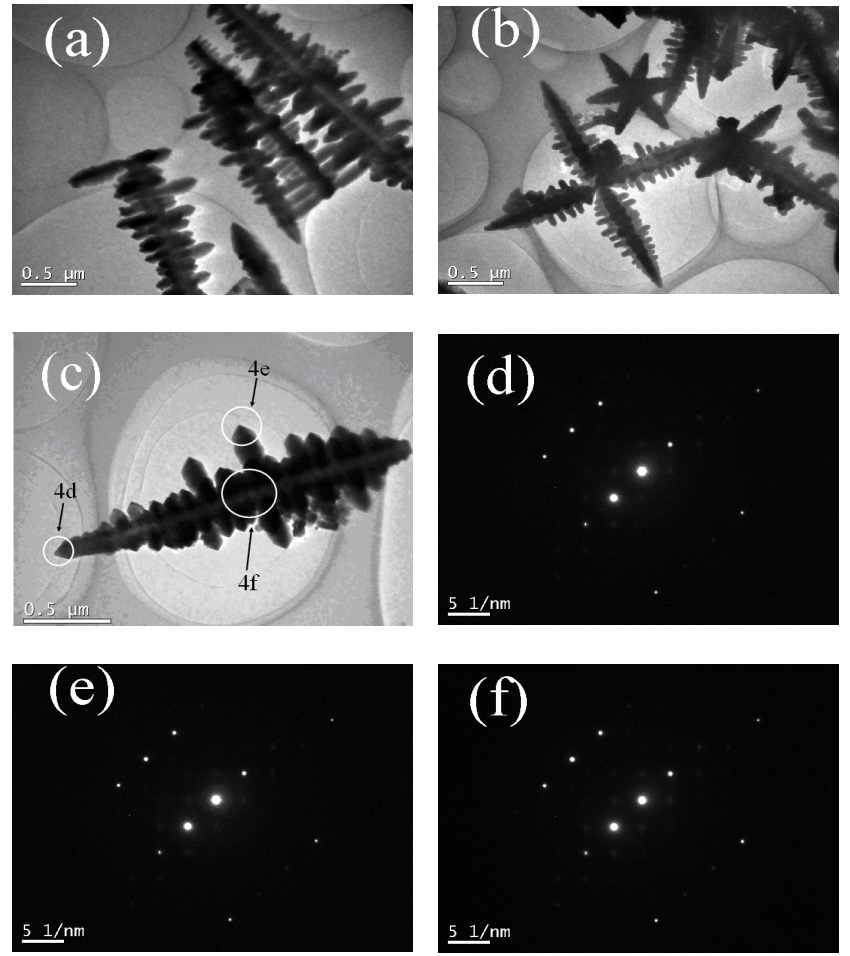

Fig. 4. TEM and ED images of the PbS dendritic nanostructures. (a) TEM images of the PbS dendritics. (b) TEM image of the "+" PbS nanostructure. (c) TEM image of the single PbS dendritic. (d) The SAED patterns taken from the long axis, the short axis and the section of the trunk, as labeled in Fig. 4d-f

In order to investigate the influence of the absence of ligand and the influence of different ligand, the following experiments were performed. The first experiment was carried out under the same reaction, but no ligand was added. It is worth to note from the SEM that different flower petal-like nanocrystal were formed (Fig. 5a-c). In the second experiment, with the use of $\mathrm{CTAB}$, well-defined $\mathrm{PbS}$ dendritic nanostructures can be obtained, which is similar to the use of TPPB (Fig. 5d). After the determination of a preferred crystalline phase during the initial stage, the subsequent growth stage strongly governs the final architecture of the nanocrystals through the delicate balance between the kinetic growth and thermodynamic growth regimes. There are at least four different parameters ( $\Delta \mathrm{G}$, time, temperature, ligand) which can influence the growth pattern of nanocrystals. The intrinsic surface of the crystallographic face of the seed is also important, since the kinetic
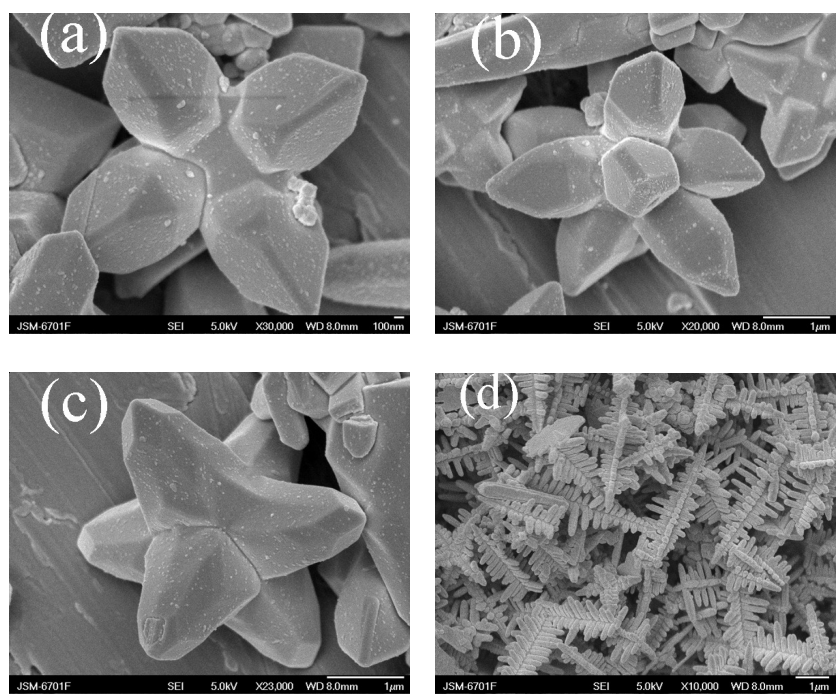

Fig. 5. (a-c) SEM images of PbS prepared in the absence of TPPB. (d) $\mathrm{SEM}$ images of $\mathrm{PbS}$ prepared with the use of $\mathrm{CTAB}$

energy barrier $(\Delta G)$ is inversely proportional to the surface energy. These surface properties can also be tailored by the types and the amounts of adsorbing organic ligand present ${ }^{21}$. By delicately balancing and controlling of these parameters, it is possible to control the shape of novel nanocrystals. It has been reported that the use of binary of capping molecular such as trioctylphosphine oxide and hexylphosphonic acid is useful for its ability to generate shape anisotropy in CdSe and also for its intrinsic hexagonal structure ${ }^{1}$.

The growth mechanisms of $\mathrm{PbS}$ crystals have been well investigated in several papers. It has been shown that such novel morphologies such as dendritic and star-shaped structures occur through preferential growth of the truncated octahedronshaped $\mathrm{PbS}$ seed $<100>$ directions ${ }^{22}$. In such a case, the presence of surfactant must be taken into consideration, for it has been reported that only star-shaped $\mathrm{PbS}$ crystals with eight < $111>$ oriented arms are produced in the absence of surfactants under similar conditions ${ }^{23}$. Therefore, it is believed that the TPPB acts as a structure directing agent. The role of hydrothermal treatment is on the one hand related to a continuous supply of the energy needed for production and assembly of the $\mathrm{PbS}$ crystals and on the other hand to a most uniform approach of supplying ions to all faces of octahedron crystal nuclei, so that the growth takes place on each face simultaneously. During the experiment process, all the TPPB molecules have the equal opportunity to prefer binding to specific crystal faces and thus act as a soft template to guide the growth of crystal until the reagents are exhausted. With the longer reaction time, the TPPB may terminate the role of soft template and the black $\mathrm{PbS}$ semiconductor nanocrystal was obtained. After the autoclave was naturally cooled, it can be seen that the PTFE tube was aggregated with white needle-like crystals (Fig. 6). This phenomenon may be attributed to the fact that the TPPB slightly soluble in cold water and soluble in hot water. After the reaction finished, some TPPB stays the surface of semiconductor in the form of chemisorbed or physical adsorption and the residual TPPB experiences the process of recrystallization. At the bottom of the white needle-like crystals is black PbS nanocrystal. 

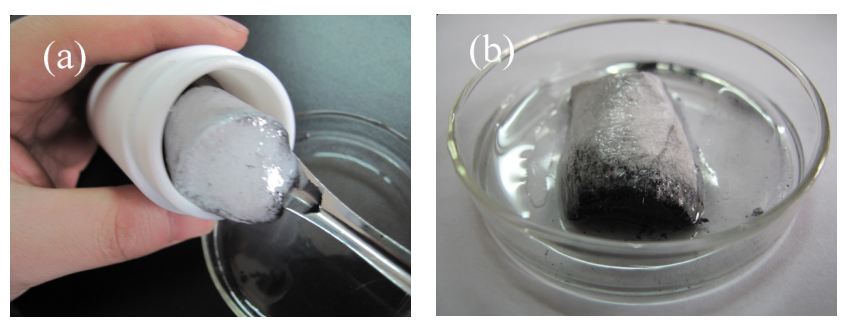

Fig. 6. Photo of experiment phenomenon

All semiconductors often possess unsatisfied dangling bonds at the surfaces, which give rise to surface states in the band gap. The preparation of semiconductor nanocrystals by the method of colloidal chemistry usually involves capping of the nanocrystal surface with stabilizing ligands which fulfill various functions, including the passivation of dangling bonds at the surfaces. Lead nanoparticles ( $c a .15 \mathrm{~nm}$ in size) were synthesized with the fatty acid (oleic acid) as a surfactant ${ }^{24}$. The results indicated that oleic acid gets chemisorbed as a carboxylate on the lead nanoparticle surfaces and the two oxygen atoms in the carboxylate are coordinated symmetrically to the $\mathrm{Pb}$ atoms, leading to the formation of the covalent $\mathrm{Pb}-\mathrm{O}$ bond. It is well-known that surface energies associated with different crystallographic planes are usually different and a general sequence can be elucidated as $\gamma\{111\}<\gamma\{100\}<$ $\gamma\{110\}^{25}$. In this experiment, capping molecules such as TPPB can selectively stabilize the $\{111\}$ faces, since their ionic head groups can strongly interact with the charged $\{111\}$ faces rather than the uncharged $\{100\}$ faces, which contain mixed $\mathrm{Pb} / \mathrm{S}$. Thus the interaction between $\mathrm{Pb}$ and $-\mathrm{P}\left(\mathrm{C}_{6} \mathrm{H}_{5}\right)_{4}+$ ions can significantly elevate the activation energy of the $\{111\}$ faces, leading to relatively greater accelerated growth on the $\{100\}$ faces relative to the $\{111\}$ faces. Therefore, TPPB plays the role of capping agent in the nucleation and growth of $\mathrm{PbS}$ crystals and the critical factor determining architectural features of the $\mathrm{PbS}$ nanocrystals. The surface structure of organically capped $\mathrm{PbS}$ nanocrystals using synchrotron radiation excited corelevel photoelectron spectroscopy has been studied ${ }^{26}$. The results indicate that the trioctylphosphine ligands passivate only the surface $\mathrm{S}$ sites while oleic acid ligands appear to bind mainly to $\mathrm{Pb}$ sites. The electronic structure of TPPB, with its tertiary $\mathrm{P}$ and the trioctylphosphine ligand are significantly different in terms of bonding orbitals. Further investigation will be focused on the photoelectron spectroscopic investigation a of chemical bonding in TPPB stabilized PbS nanocrystals.

\section{Conclusion}

In summary, well-defined $\mathrm{PbS}$ dendritic nanostructures were prepared with tetraphenylphosphonium bromide as a ligand via simple hydrothermal process. The $\mathrm{PbS}$ dendritic nanostructure was characterized by SEM, XRD and HRTEM,
ED. A possible mechanism for the formation of $\mathrm{PbS}$ dendrites has been proposed. It has been found that TPPB plays important role in the formation of well-defined $\mathrm{PbS}$ dendritic nanostructures. To the best of our knowledge, this is the first time that the TPPB was used as a ligand or stabilizer to synthesis semiconductor nanomaterials via simple hydrothermal process. The TPPB may have a bright future in controlling synthesis various morphology semiconductor nanocrystals.

\section{ACKNOWLEDGEMENTS}

The authors thank the "Western Light" Talents Training Program of CAS and the Solar Action Plan of CAS (No. 1731012394) for financial support.

\section{REFERENCES}

1. X.G. Peng, L. Manna, W.D. Yang, J. Wickham, E. Scher, A. Kadavanich and A.P. Alivisators, Nature, 404, 59 (2004)

2. L. Bakueva, G. Konstantatos, L. Levina, S. Musikhin and E.H. Sargent, Appl. Phys. Lett., 84, 3459 (2004).

3. M. Nirmal and L.E. Brus, Acc. Chem. Res., 32, 407 (1999).

4. D. Kuang, A. Xu, Y. Fang, H. Liu, C. Frommen and D. Fenske, Adv. Mater., 15, 1747 (2003).

5. S. Wang and S. Yang, Langmuir, 16, 389 (2000).

6. S.M. Lee, Y.W. Jun, S.N. Cho and J.W. Cheon, J. Am. Chem. Soc., 124, 11244 (2002).

7. E. Leontidis, M. Orphanou, T. Kyprianidou-Leondidou, F. Krumeich and W. Caseri, Nano Lett., 3, 569 (2003).

8. Y. Ni, H. Liu, F. Wang, Y. Liang, J. Hong, X. Ma and Z. Xu, Cryst. Res. Technol., 39, 200 (2004).

9. D. Kuang, A. Xu, Y. Fang, H. Liu, C. Frommen and D. Fenske, Adv. Mater., 15, 1747 (2003).

10. Y. Ni, X. Wei, J. Hong and X. Ma, Cryst. Res. Technol., 41, 885 (2006).

11. Y.K.A. Lau, J.C. Davin, J.B. Matthew and J. Song, J. Am. Chem. Soc., 131, 16461 (2009).

12. Z.P. Qiao, Y. Zhang, L.T. Zhou and Q. Xire, Crystal Growth Design, 7, 2394 (2007).

13. F. Gao, Q. Lu, X. Liu, Y. Yan and D. Zhao, Nano Lett., 12, 743 (2001).

14. N. Wang, X. Cao, L. Guo, S. Yang and Z. Wu, ACS Nano, 2, 184 (2008).

15. R. Xie, D. Li, D. Yang and M. Jiang, J. Mater. Sci., 42, 1376 (2007).

16. N. Zhao and L. Qi, Adv. Mater., 18, 359 (2006)

17. D. Kuang, A. Xu, Y. Fang, H. Liu, C. Frommen and D. Fenske, Adv. Mater., 15, 1747 (2003).

18. J. Xiang, H. Cao, Q. Wu, S. Zhang and X. Zhang, Crystal Growth Design, 8, 3935 (2008).

19. B.L. Wehrenberg, C. Wang and P. Guyot-Sionnest, J. Phys. Chem. B, 106, 10634 (2002).

20. K.A. Dick, K. Deppert, M.W. Larsson, T. Mårtensson, W. Seifert, L.R. Wallenberg and L. Samuelson, Nat. Mater., 3, 380 (2004).

21. S.M. Lee, S.N. Cho and J. Cheon, Adv. Mater., 15, 441 (2003).

22. S.M. Lee, W.W. Jun, S.N. Cho and J. Cheon, J. Am. Chem. Soc., 124, 11244 (2002).

23. G. Russell, P. Henrichs, J. Hewitt, H. Grashof and M. Sandhu, Macromolecules, 14, 1764 (1981)

24. N. Wu, L. Fu, M. Su, M. Aslam, K.C. Wong and V.P. Dravid, Nano Lett., 4, 383 (2004)

25. Z.L. Wang, J. Phys. Chem. B, 104, 1153 (2000).

26. A. Lobo, T. Mo1ller, M. Nagel, H. Borchert, S.G. Hickey and H. Weller, J. Phys. Chem. B, 109, 17422 (2005). 